\section{Antimatter and the Moon}

SIR - The recent measurement of the shadow of the Moon in charged cosmic rays with energies in the $10 \mathrm{TeV}$ energy region $^{1}$ shows a displacement of $0.15^{\circ}$ from the Moon's position; as pointed out previously $^{2}$ the Earth-Moon system acts as an ion spectrometer and can be used to differentiate matter and antimatter in the cosmic radiation. The direction and magnitude of the shift in this Tibetan airshower experiment is in agreement with the hypothesis that most of the cosmic radiation at this energy is ter). Although the cosmic radiation does show an anomalously high flux of antiprotons at energies of $1-10$ $\mathrm{GeV}$ (ref. 3), there is no direct experimental evidence of the nature of the cosmic radiation at energies above $20 \mathrm{GeV}$; in principle ponent could be antimatter.

On the assumption that the Universe is baryon symmetric and that higherenergy cosmic rays are of extragalactic origin, Stecker and Wolfendale ${ }^{4}$ have shown that the observed ratio of $\bar{p} / \mathrm{p}$ below $20 \mathrm{GeV}$ can be explained; at energies of 10 is that it rises with energy to as much as $20 \%$ above $10 \mathrm{TeV}$.

The significance of the Moon shadow detection is at the 7.10 level (Fig. 1); the displacement of the centroid of the shadow image from the known position of the Moon is $(0.16+0.11 /-0.09)^{\circ}$ to the west and $(0.02+0.07 /-0.09)^{\circ}$ to the south. The calculated shift of the image by the geomagnetic field is estimated to be $0.15^{\circ}$ to the west ${ }^{1}$ for the mode energy of protons at the zenith angle of the observations.

If the cosmic radiation was composed

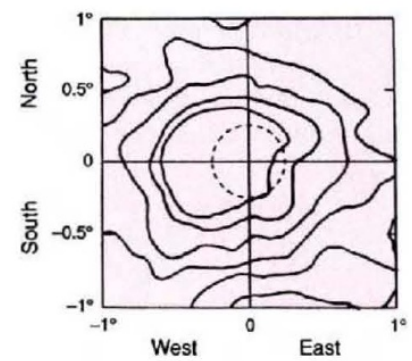

Fig. 1 Distribution of arrival directions of air showers in a box $2^{\circ} \times 2^{\circ}$ centred on the position of the Moon as recorded in the Tibet air shower experiment ${ }^{1}$. composed of protons (matall of this high energy com$\mathrm{GeV}$ this ratio is $0.1 \%$, and the prediction entirely of antiprotons, the shadow would be displaced to the east by the same amount; a mixed composition would result in an elongated or displaced image. We have simulated the Moon image for various mixtures of matter and antimatter $00 \%$ PBAR

$1^{\circ} \quad 30 \%$ PBAR
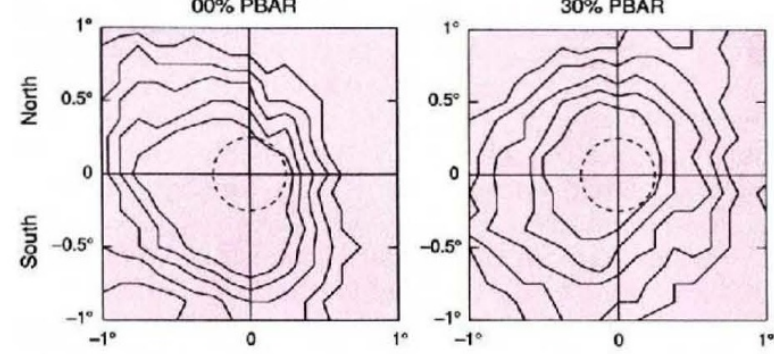

$50 \%$ PBAR
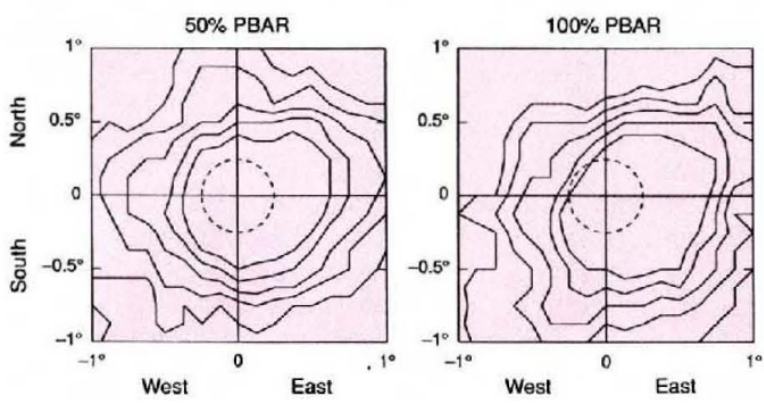

East
Fig. 2. Simulations of the arrival directions of a similar number of events assuming the composition $(\bar{p} / p+\bar{p})$ is 0,30 , 50 and $100 \%$.

assuming a uniform magnetic field deflection of $30 \mathrm{mrad} E^{-1}(\mathrm{TeV})$ and using a similar analysis procedure to that used in the Tibetan experiment (Fig. 2). The clear displacement of the measured image to the west (matter) and the lack of any significant elongation in the east-west direction agrees with the hypothesis that the bulk of the cosmic radiation at these energies is composed of protons. Although it is difficult to arrive at a quantitative limit without a detailed modelling of the Tibet experiment, a conservative figure (based on a visual comparison of Figs 1 and 2) is that the antimatter component is less than $30 \%$; this limit is not sufficient to challenge the baryon symmetric model ${ }^{4}$ but does show that all the high-energy cosmic rays cannot be antimatter.

At energy thresholds of $1 \mathrm{TeV}$ the offset is as much as $1.5^{\circ}$. More precise measurements of the moon shadow and hence of the $\bar{p} / \mathrm{p}$ ratio in the $1-10 \mathrm{TeV}$ region will soon be forthcoming from the

1. Amenomori, M. et al. Proc. $23 r d$ int . Cosmic Ray Conf. 4 , 351-354 (Calgary, 1993)

2. Urban, M. etal. Nucl. Phys. (Proc. Suppl. 14B), 22's-236 (1990)

3. Stephens, S. A. \& Golden, R. L. Space Sci. Rev. 46, 31 (1987).

4. Stecker, F. W. \& Wolfendale, A. W. Nature 309, 37-38 (1984).

5. Appollinari, G. etal. Nucl. Inst. Meth. A263, 255 (1988).
ARTEMIS $^{2}$ and CLUE $^{5}$ experiments using the atmospheric Cherenkov technique in the ultraviolet.

M. Chantell

\section{T. C. Weekes}

Hanvard-Smithsonian Center for

Astrophysics,

Whipple Observatory,

PO Box 97, Amado,

Arizona 85645-0097, USA

X. Sarazin

M. Urban

LPNHE, Ecole Polytechnique/

CNRS-IN2P3,

91128 Palaiseau, France

\section{Ancient rituals in Gabon}

SIR - During a palaeontological survey of caves in Gabon, West Africa, in 1992, we discovered evidence of past human presence in Paouan cave, near Lastoursville ${ }^{1}$, well beyond the limit of light penetration. A sondage in the sediments comprising the floor of a vast chamber revealed the presence of archaeological levels which contained abundant stone tools and charcoal. We examined samples of charcoal by microscope to determine the plant species present, and analysed others by the ${ }^{14} \mathrm{C}$ method to obtain evidence of the ages of the layers. The earlier of the two levels is $5,570 \pm 70$ years before present, the later is $4,000 \pm 70$ years before present.

The stone tools from the lower level consist of hammer stones, flakes, cores, blades and rarer triangular points worked in black to grey jasper and milky quartz. The assemblage as a whole resembles the Tschitolean industry which is widespread in Zaire, Congo and Gabon ${ }^{2}$. Both types of raw material consist of water-worn pebbles introduced into the dolomite cave by humans.

Among more than 80 charcoal fragments identified, only four species of plant are represented, which indicates that the choice of plants carried into the cave was highly selective, considering that the forest surrounding the cave is extremely diverse (more than 1,000 woody plant species grow in Gabon). The four plant species found in archaeological context in Paouan cave are well known to local inhabitants, all of them having magicalritual properties. The tree Pterocarpus tinctorius yields a red powder used to colour the body, especially during ceremonies such as initiation rites. Copaifera resin (copal) is used to manufacture torches, the tree itself considered as the 'king of trees' by many people of the African tropical forest. Combretum yields extremely hard wood often used to make batons and clubs, the wood being said to impart "aggressive" qualities to the holder. Finally, the liana Strophanthus is used 